\title{
A MICROTEXTURE INVESTIGATION OF SIDE- BRANCHED FATIGUE CRACKS IN 8090 Al-Li SHEET
}

\author{
VALERIE RANDLE \\ Department of Materials Engineering, University College of Swansea, Singleton \\ Park, Swansea SA2 8PP, U.K.
}

(2 January 1993)

\begin{abstract}
Electron back-scatter diffraction has been used to assess the microtexture of regions of $8090 \mathrm{Al}-\mathrm{Li}$ sheet both where side-branched fatigue cracking has occurred and in control areas unaffected by cracking. The microtextures did not reflect the macrotexture as measured by $\mathrm{X}$-rays, indicating inhomogeneous distributions of orientations. The microtexture of the "cracked" grains was complex and was deconvoluted to reveal several components dominated by the S-texture. The principal difference between "cracked" and "control" grains was that for the former the four crystallographically related $S$ variants were intimately mixed on an individual grain scale whereas in the latter was mainly a single variant in a particular area. The results are discussed in terms of both recovery and recrystallisation mechanisms.
\end{abstract}

KEY WORDS Aluminium-lithium sheet alloy, fatigue crack growth, electron back-scatter diffraction, orientation clustering.

\section{INTRODUCTION}

Aluminium-lithium alloys have been developed to provide a lower density alternative for conventional aerospace alloys. The addition of lithium to aluminium brings both a density improvement and an increase in modulus. The 8090-T3 damage tolerant sheet examined in this work is intended for fuselage skinning applications where a high degree of resistance to fatigue crack growth is required.

On a microscopic scale, a fatigue crack follows a tortuous path with deviations on a scale similar to the grain size away from the general growth direction which is perpendicular to the applied load. There is also a significant degree of side-branch cracking. The rough crack surface results in a high degree of closure during the fatigue cycle and is partly responsible for the improved fatigue crack growth rate performance of this material. In addition to this microscopic deviation, in some tests the fatigue crack turns through an angle of about $70^{\circ}$ towards the loading direction and is then stable in this direction. Once macroscopic deviation occurs the crack becomes smooth and side branching is a minimum.

The fact that this macroscopic deviation is worse in certain testpiece orientations suggests an influence due to crystallographic texture and this has received much scrutiny. However comparison using ODF techniques of deviant and 
non-deviant samples has so far failed to reveal a conclusive trend. The current focus aims to use microtexture techniques to elucidate the cause of the variable deviation performance. The work reported here sets out to determine the local texture adjacent to branched cracks to assess whether local areas of preferred orientation exist. If so, future work may extend to microtexture determination local to the deviated crack.

Previous macrotexture ODF information indicates that for the $\frac{1}{2}$-thickness plane the predominant recrystallised texture is Goss, with some cube. Other deformation textures are present, namely $\{110\}\langle 112\rangle$ (brass), $\{4,4,11\}\langle 8,11,11\rangle$ (Taylor) and $\{123\}\langle 634\rangle$ or $\{123\}\langle 412\rangle$ (S) (Bowen et al., 1991). Near the sheet surface the cube texture becomes predominant.

\section{EXPERIMENTAL}

The composition of the material examined is shown in Table 1 . A specimen $420 \times 160 \times 20 \mathrm{~mm}$ with a $5 \mathrm{~mm}$ notch machined in the centre was fatigued at constant applitude with an $R$ ratio of 0.1 . The applied load was $30.3 \pm 24.8 \mathrm{kN}$ and deviation occurred when the applied stress intensity, $\mathrm{K}$, was $31.1 \mathrm{MPa} \vee \mathrm{m}$. A specimen was then sawn from the test piece as shown in Figure 1 and polished to $\frac{1}{2}$ thickness using standard metallographic techniques. The final polishing step used colloidal silica.

Orientations of 428 grains from a total of 55 cracks and 3 "control" areas were measured using electron back-scatter diffraction (EBSD) in a scanning electron microscope (SEM). The principles and practice of EBSD are expounded in detail elsewhere (Randle, 1992; Dingley and Randle 1992) and so are not described here. Briefly, the technique allows single orientations from regions of crystal with dimensions approximately $200 \times 200 \times 20 \mathrm{~nm}$ to be measured and related to their position in the microstructure via observation in the SEM. The application of EBSD in this case was to measure a population of spatially specific orientations from grains through which a side-branched crack was associated, and compare this orientation population with those measured from uncracked, control grains. The EBSD method is particularly advantageous for the study of cracked material since specimen preparation for transmission electron microscopy is not possible. All data sets i.e. those from the cracked and uncracked regions were recorded from the same polished plane in the specimen i.e. the $\frac{1}{2}$-thickness plane as shown in Figure 1.

Table 1 Composition in weight $\%$ of the material investigated.

\begin{tabular}{llllllll}
\hline$S i$ & $F e$ & $C u$ & $M g$ & $T i$ & $L i$ & $Z r$ & $A l$ \\
\hline 0.04 & 0.04 & 1.10 & 0.76 & 0.02 & 2.28 & 0.06 & bal. \\
\hline
\end{tabular}




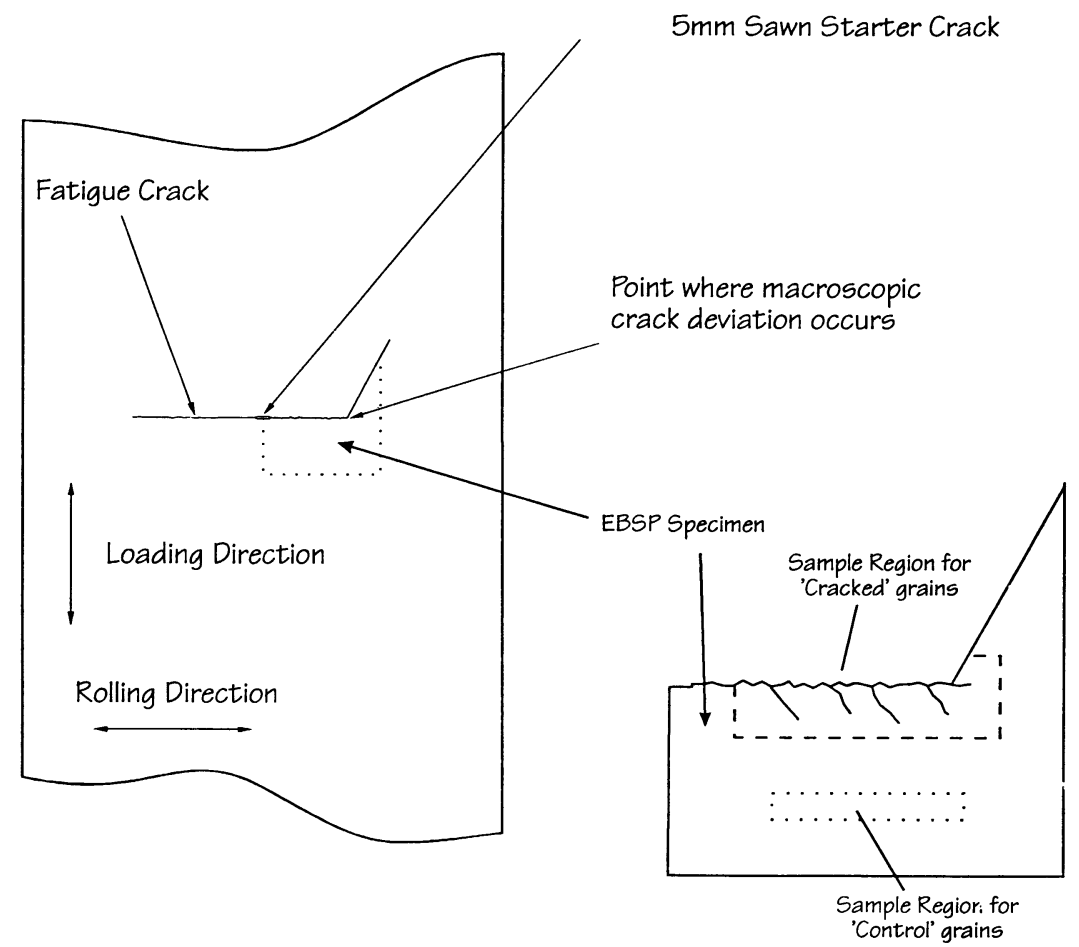

Figure 1 Schematic illustration showing the macroscopic geometry of the test piece and the EBSD specimen in relation to cracking and the two sample regions, "cracked" and "control" grains (Courtesy of K. Gatenby).

\section{RESULTS AND DATA PROCESSING}

\subsection{Microstructure and Crack Morphology}

Figure 2a shows an example of a side-branched crack (throughout the remainder of this paper, "crack" will refer to side-branched cracks unless otherwise specified). Previous metallography had revealed an equiaxed grain structure, and in terms of grain diameters crack lengths varied from 2 to 18, with most cracks traversing 6-10 grains. Cracks followed an erratic path, with direction changes occurring both within grains and also at grain boundaries. In some long cracks subsidiary branching was evident. There was also some intergranular component to crack paths, as determined by EBSD pattern changes, although this was minor and most cracks ran intragranularly. The crack direction deviation at grain boundaries infers a crystallographic character to the crack plane. Examples of all the above-mentioned features of crack morphology are indicated on Figures $2 b$, c, d. Individual orientation classifications are also indicated on these figures, and are discussed in section 3.3 and section 4. Diffraction patterns from areas nearest to the primary crack were usually very degraded because of strain in those regions. 

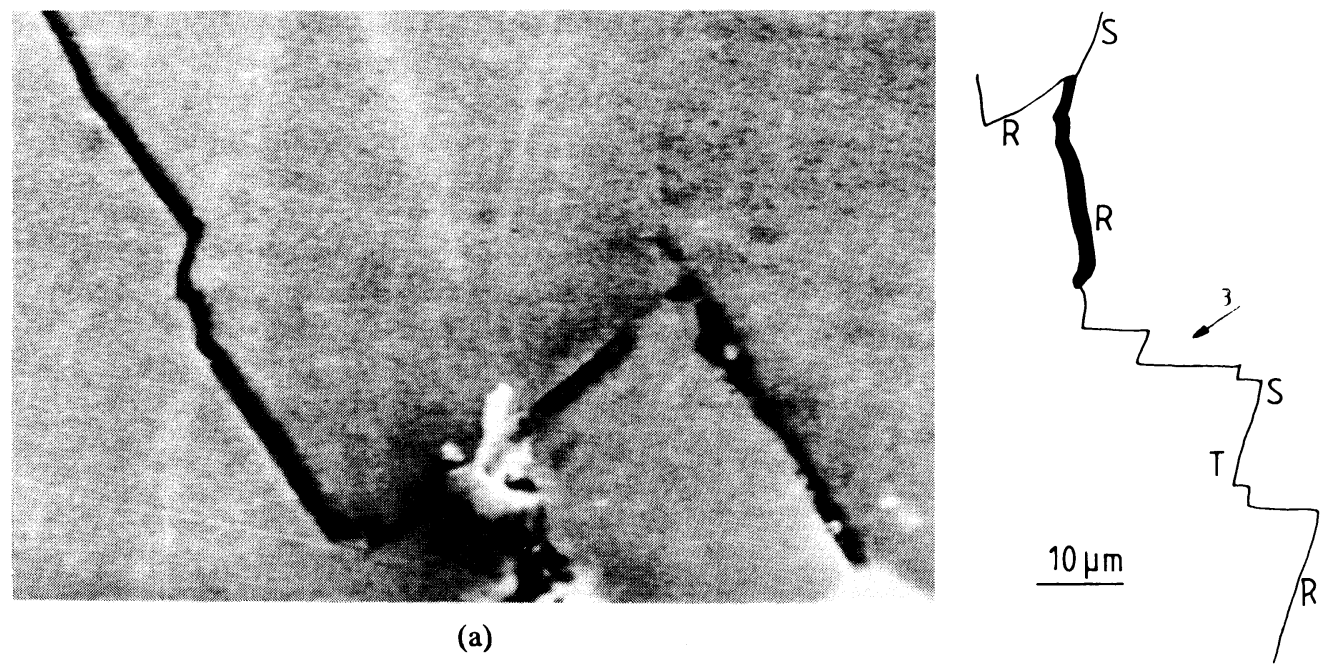

(a)

(c)
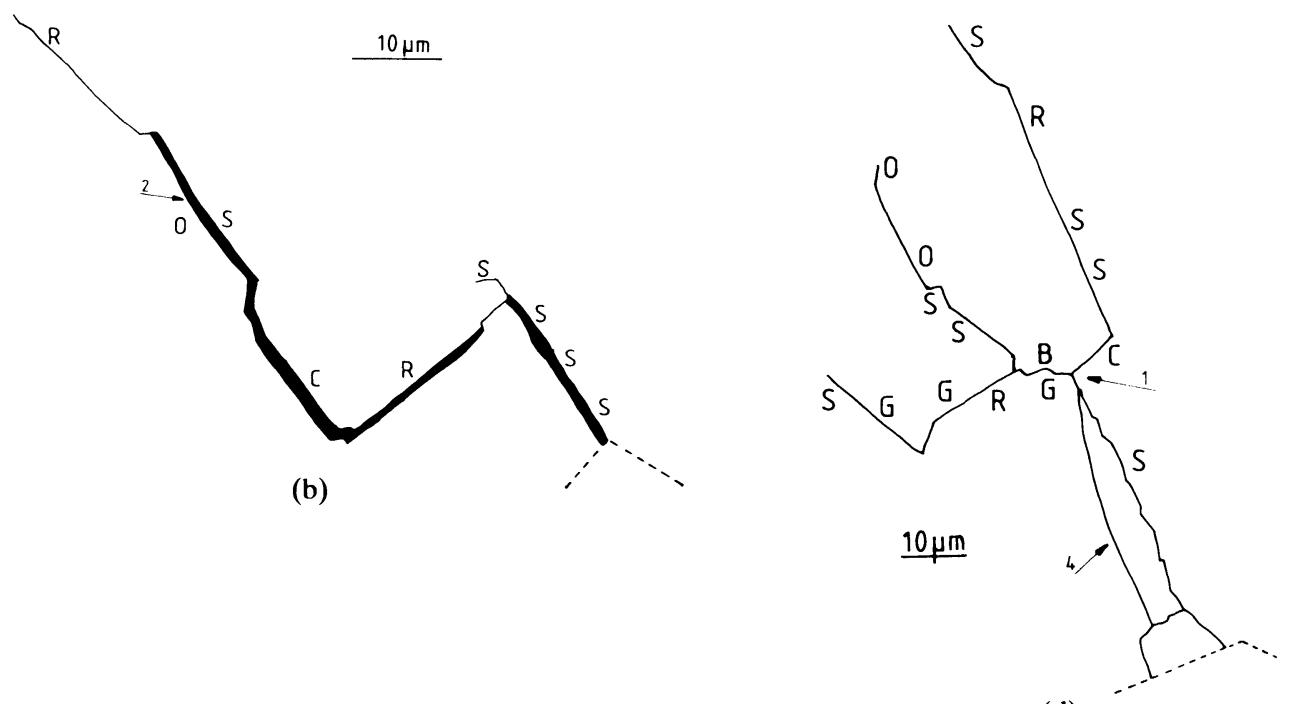

(d)

Figure 2 (a) micrograph showing the typical morphology of a side-branched crack. (b), (c), (d) The orientation group of each grain is shown on schematic illustrations of three cracks. (b) is the crack shown in (a). The orientation groups are labelled as follows: $S(1,2,3,4)\{123\}\langle 412\rangle, G\{110\}\langle 001\rangle$, $\mathrm{C}\{100\}\langle 001\rangle, \mathrm{R}\{013\}\langle 001\rangle, \mathrm{T}\{4,11,11\}\langle 8,11,11\rangle, \mathrm{B}\{110\}\langle 112\rangle$, O other orientations. Numbered arrows indicate one example each of (1) multiple crack branching, (2) intergranular crack path, (3) crack direction change within a grain, (4) region where the EBSD pattern is very diffuse. The dashed line indicates the main crack. 


\subsection{Pole Figure Representation of the Microtextures}

The use of pole figures to display orientations degrades the available EBSD information, since a pole figure is a two-dimensional projection used for displaying crystallographic directions or plane normals whereas grain orientations measured using EBSD contain the full, three dimensional orientation of the grain. However, it is sometimes convenient to use a pole figure to display microtexture data (either as individual data points or smoothed to contours) for the following reasons:

1. Pole figures are familiar because of their traditional use for (macro)textures which have been generated using $\mathrm{X}$-rays;

2. Direct comparisons can be made between macrotexture and microtexture data;

3. Relatively small microtexture data sets, i.e. less than about 200 data points, tend to get "lost" in a three dimensional sectionalised data display format such as Euler space or Rodrigues-Frank space and, unless the texture is very sharp, visual interpretation of the distribution is difficult. In this situation contour lines may improve the representation of the complete orientation distribution.

Furthermore, it is convenient to maintain for microtexture the "ideal orientation" terminology familiar to the interpretation of macrotexture, i.e. $(h k l)[u v w]$ where $h k l$ is the rolling plane and $u v w$ the rolling direction. For the data analysed here an $(h k l)\langle u v w\rangle$ value was assigned to individual orientations on the basis of a $\pm 15^{\circ}$ spread.

The microtextures of cracked and control grains are shown in pole figure format in Figures 3 and 4 respectively. It is particularly striking that the total distribution in Figure 3a is different from the total distribution of the control grains, Figures $4 a, b$ and $c$. This observation highlights immediately the advantages of the microtexture approach: the small-scale orientation sampling is able to show that there is a difference between the textures of cracked and uncracked regions. These differences are masked by the averaging effect of macrotexture as determined by X-rays. The microtextures can be deconvoluted into specific components, which for the cracked grains is shown in Figures $3 b-3 h$. These specific components account for $91 \%$ of the total orientations measured.

The control grains were sampled in 3 linear scans of 50 grains each, parallel to the rolling direction. The major component, $59 \%$ of the total sample, is the S-texture $\{123\}\langle 412\rangle$. There are also minor components of Goss, $\{110\}\langle 001\rangle$ $(17 \%)$, and $\{013\}\langle 100\rangle(11 \%)$. The latter component is a "rotated cube" orientation where the rotation is about the normal to the rolling plane. The microtexture from the second control set, shown in Figure $4 \mathrm{~b}$, is a mirror-related (twin) variant of $\{123\}\langle 412\rangle$ to that from control areas 1 and 3 , Figures $4 \mathrm{a}$ and $4 \mathrm{c}$ respectively.

The texture of the cracked grains is more complex than that of the control grains; its components are $48 \%\{123\}\langle 412\rangle, 15 \%\{110\}\langle 001\rangle, 7 \%\{100\}\langle 001\rangle$ (the cube texture) and some other minor components, namely $\{013\}\langle 100\rangle$, $\{4,4,11\}\langle 8,11,11\rangle,\{111\}\langle 110\rangle$ and $\{110\}\langle 112\rangle$. In the data reported here most of the S-oriented grains are better described as $\{123\}\langle 412\rangle$ rather than $\{123\}\langle 634\rangle$; for convenience the few grains which are close to $\{123\}\langle 634\rangle$ are subsumed within the $\{123\} 412\} \mathrm{S}$ category. Table 2 shows the overall breakdown 

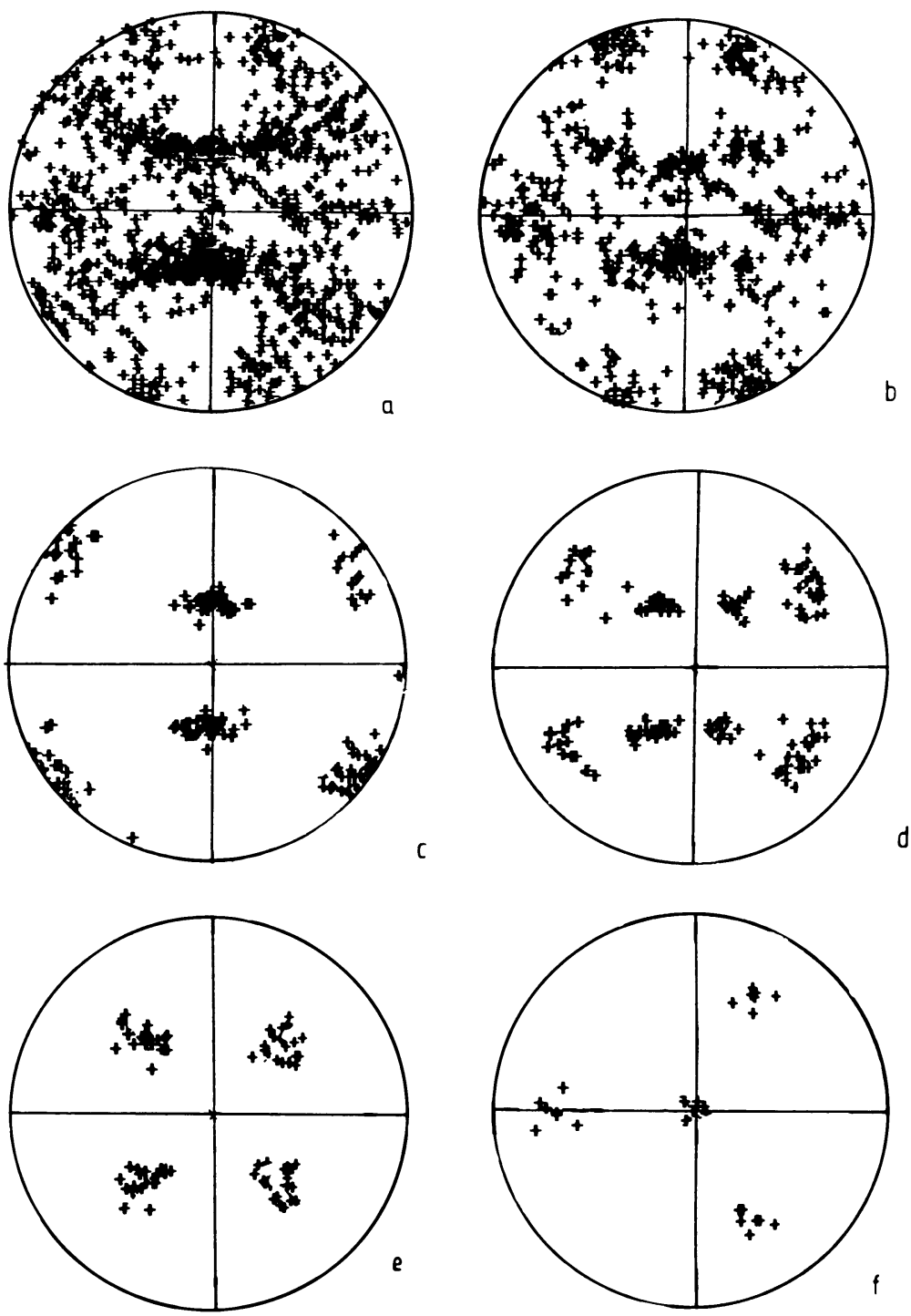

Figure 3111 microtexture pole figures of the cracked grain data set, comprising 278 grains. (a) all grains, (b) $\{123\}\langle 412\rangle$, (c) $\{110\}\langle 001\rangle$, (d) $\{013\}\langle 100\rangle$, (e) $\{001\}\langle 100\rangle$, (f) $\{111\}\langle 110\rangle$, (g) $\{4,11,11\}\langle 8,11,11\rangle$ (h) $\{110\}\langle 112\rangle$.

of the microtexture proportions for all the data. For cases where an orientation is described by more one ideal texture category, it is counted in the majority group.

The principal difference between the control and cracked grains is that for the former a single variant of $\{123\}\langle 412\rangle$ is dominant in each of the three sampled regions, either (123)[412] (S1) or its twin-related variant (213)[142]] (S2), whereas for the cracked grains the $\{123\}\langle 412\rangle$ component is approximately equally split between each of the four components of $\{123\}\langle 412\rangle$. Hence the pole 

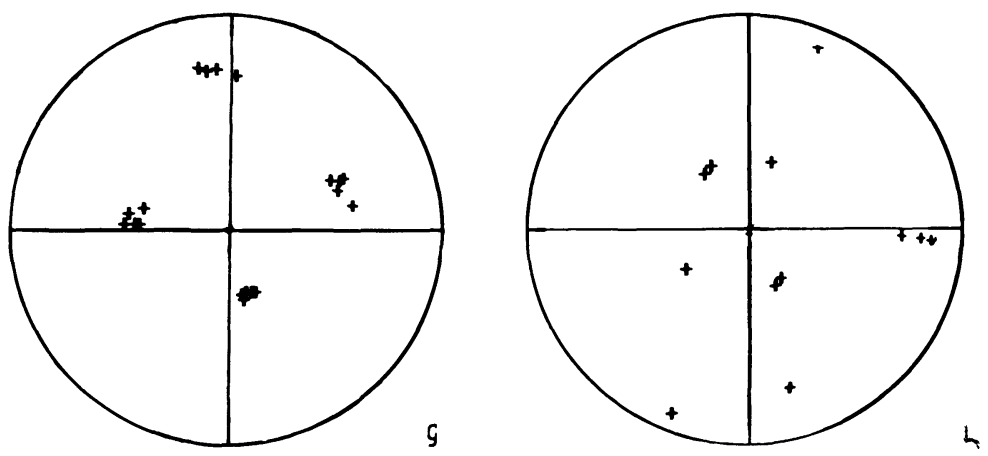

Figure 3 (continued)
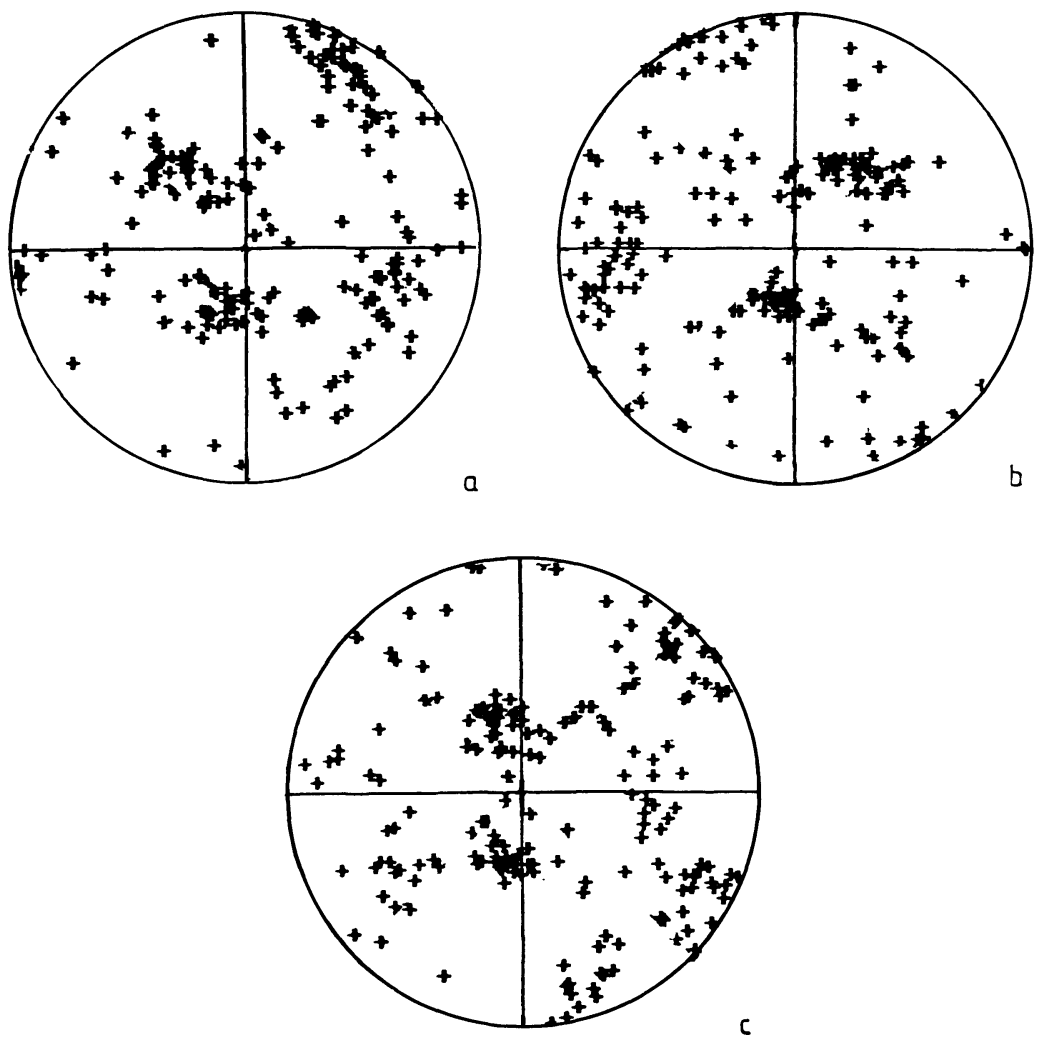

Figure 4111 microtexture pole figures from three control data sets, (a), (b) and (c), of 50 grains each. (a) and (c) are predominantly (123)[412] (S1) and (b) is (213)[142] (S2). $\{011\}\langle 100\rangle$ and $\{013\}\langle 100\rangle$ are secondary components in each pole figure. 
Table 2 Proportions (\%) of orientations (within $15^{\circ}$ ) in the sample populations of grains associated with cracks and control grains.

\begin{tabular}{lrl}
\hline Texture component & “Cracked" grains & Control grains \\
\hline$\{110\}\langle 001\rangle^{a}$ & 16 & 17 \\
$\{013\}\langle 100\rangle^{a}$ & 14 & 11 \\
$\{001\}\langle 100\rangle^{a}$ & 7 & - \\
$\{123\}\langle 412\rangle$ & 48 & 59 \\
$\{4,4,11\}\langle 8,11,11\rangle$ & 2 & - \\
$\{110\}\langle 112\rangle$ & 1 & - \\
$\{111\}\langle 110\rangle$ & 3 & - \\
\hline
\end{tabular}

a Recrystallisation textures.

figure for the cracked grains has a symmetrical appearance compared to the asymmetry of the control grains' pole figure where the microtexture is polarised towards a single variant.

\subsection{Distribution of Microtexture Components}

One of the principal advantages of microtexture determination is its ability to link the spatial and orientational characteristics of the microstructure. Orientations were measured linearly for the control data so that nearest neighbours were identified. For the cracked grains, orientations were correlated with their position in relationship to the particular crack as shown by the examples in Figure 2 .

It is a strong feature of the results that the control data consisted mainly of a single variant of the S-texture whereas all the S-texture variants, S1, S2, S3 and $S 4$, plus other texture components, were represented for the cracked grains. Furthermore, the distribution of all the texture components and variants is considerably mixed with respect to position along a crack. This is illustrated on Figure 2, where the various components are identified by letters according to the key. The designation of $S$ orientations as $S 1,2,3$ or 4 is from analysis of the orientation matrix. Although only three cracks are shown in Figure 2, the distribution (i.e. the spatially mixed nature of the texture components) is typical for the cracks sampled.

\subsection{Misorientations}

Two individual orientations can be re-expressed as a single misorientation. This concept is applied to microtexture in two ways: a statistical distribution of misorientations can be obtained from the distribution of orientations, and the spatial connectivity of orientations can be used to reveal the relationship between neighbouring or other spatially selected grains (Bunge and Weiland, 1988). In the present work, it is instructive to analyse the misorientations between contiguous grains or between selected grains e.g. S-texture components associated with the same crack. From these analyses non-random. elements such as small angle boundaries (usually indicating the same neighbouring texture variant), twinning elements and also clustering of related orientations can be identified. Illustrations of small angle or twin relationships between non-contiguous grains within the same crack are indicated on Figure 2. 
There are $9 \%$ and $30 \%$ small angle (i.e. $\leq 20^{\circ}$ ) misorientations between contiguous grains in the cracked and control data sets respectively. The higher proportion of small angle boundaries for control grains compared to cracked grains is a consequence of the predominance of the $\{123\}\langle 412\rangle$ single variant which exists in the control data sets. This reaffirms the observation in section 3.3 that the texture variants of cracked grains do not cluster. For both the cracked grains and the second set of control data (which consists mainly of an S-texture variant which is twin-related to that of the first and third sets), the small angle boundaries are distributed randomly. However, for the first and third set of control data there is evidence of clustering of small angle boundaries and other misorientations. This misorientation clustering can be demonstrated in Rodrigues-Frank space.

An effective way to display misorientations, and in this case their variation within a linear array of grains, is via the Rodrigues vector. The Rodrigues vector $(R$-vector) is defined as

$$
R=U V W \tan (\theta / 2)
$$

where $U V W$ and $\theta$ are the axis and angle of misorientation respectively. When the lowest angle solution of the 24 symmetry-related variants is selected, the $R$-vectors reside in a space known as Rodrigues-Frank (RF) space. RodriguesFrank space and the $R$-vector are described in detail elsewhere (Frank 1988; Randle, 1990). The $R$-vector provides a useful means of representing combined spatial/orientational information (e.g. Weiland, 1992). In this instance the $\boldsymbol{R}$-vector is used to represent misorientations between neighbouring grains; it could similarly be used to represent orientations.

Figure 5 shows the projection of $R$-vectors in the $R_{1} R_{2}$ plane, with $R_{3}$ perpendicular to the plane of the paper, for misorientations between the 50 grains in the first control data set. There are similar clustering tendencies in the third control data set, but the trend is less pronounced in the second set where the texture is predominantly $S 2$, i.e. twin related to $S 1$, rather than $S 1$ as in the first and third control sets. In Figure 5 a the $R$-points (i.e. tips of the $R$-vectors) are shown for all 49 misorientations, and in Figure $5 b$ the $R$-vector for each individual misorientation is represented sequentially in RF space. The $R_{3}$ component is depicted qualitatively here by the thickness of the arrow defining the $R$-vector. For most of the misorientations $R_{3} \approx 0$. These are shown with small symbols on Figure 5a or a narrow line on Figure 5b. The $R_{1} R_{2} R_{3}$ axes of RF space are parallel to misorientation axes $100,010,001$ respectively. The three components of the $R$-vector are derived from $\theta$ and $U V W$ with $U V W$ all positive and $U>V>W$. The clustering effect of misorientations is evident from the linear sequence of orientations in Figure 5. The length of the $R$-vector is proportional to the misorientation angle, and thus it is clear that there is a trend for misorientation angles of a similar magnitude, particularly small angle boundaries, to be clustered together. The misorientation axis is represented by the direction of the $R$-vector, and it is further evident that similar misorientations occur together, e.g. misorientations 10/11, 11/12, and 43/44, 44/45, 45/46. Conversely, there is a random arrangement of misorientations for grains 23 through 30 . There is a twin relationship between grains 10 , near (123)[ [412], and 11, (111)[101] , which is shown in pole figure format in Figure 6. 


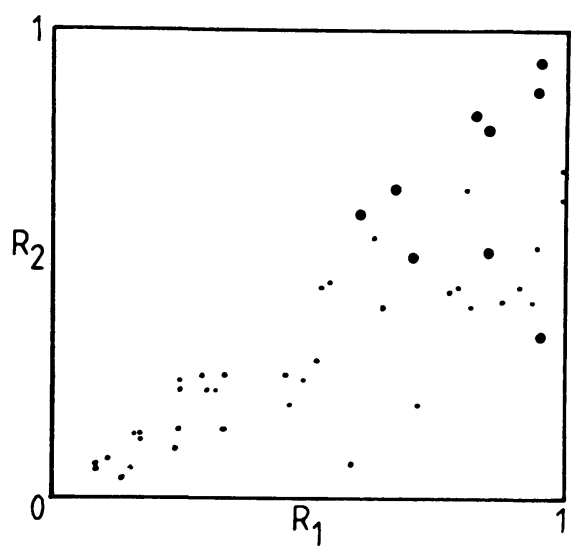

(a)
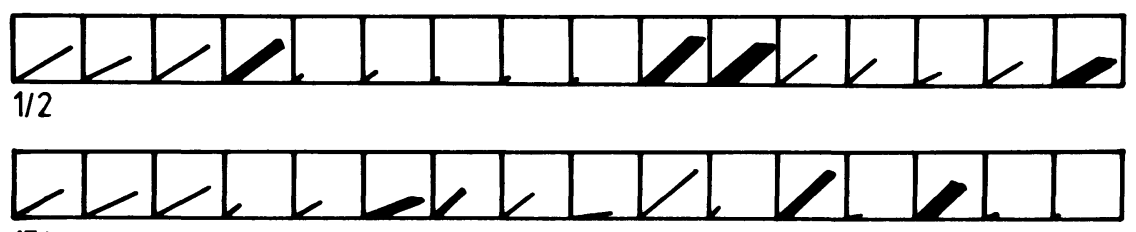

$17 / 18$

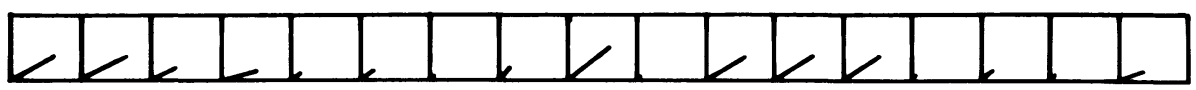

$33 / 24$
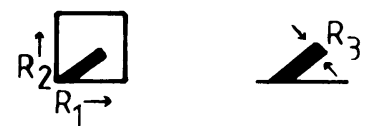

(b)

Figure 5 (a) Rodrigues points (the tips of $R$-vectors) for misorientations between neighbouring grains in the first control data set. The points are projected onto the $R_{1} R_{2}$ plane of RF space, labelled in units of the fundamental zone. Small and large symbols indicate $R$-vectors having $R_{3}<0 \cdot 1$ and $R_{3}>0 \cdot 1$ respectively. (b) Rodrigues vectors for misorientations between sequential neighbouring grain pairs in the first control data set. The $R_{1}$ and $R_{2}$ components correspond to orthogonal directions in the plane of the paper and $R_{3}$ is represented qualitatively by the thickness of the line depicting the vector. Rodrigues vectors for contiguous grain pairs are arranged sequentially i.e. grains $1 / 2,2 / 3,3 / 4$ etc. in order to demonstrate the linear grouping of misorientations, particularly for the case small angle misorientations.

\subsection{Comparison with the Macrotexture}

Although previous ODF analyses have shown that the Goss texture predominates at the mid-thickness plane, the present data indicates that there is an inhomogeneous distribution of texture on the scale of the same population size, i.e. 50-200 grains, since the major component is not Goss but rather the S-texture. The S-texture, also Taylor and Brass textures, are components of the deformation texture. However, the grain structure is equiaxed throughout, which indicates that there has been some retention of these deformation textures during a 


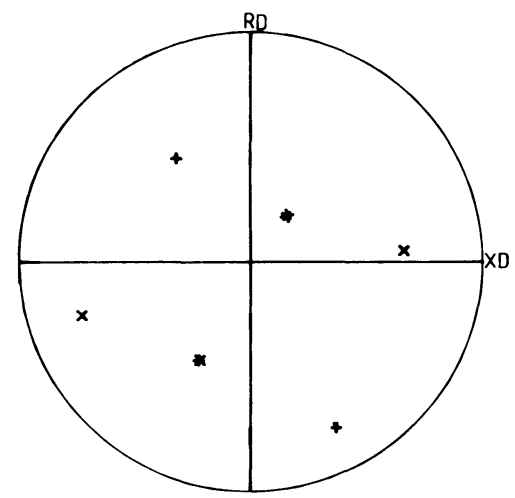

Figure 6200 microtexture pole figure for two grains in the first control data set, demonstrating their twinned relationship.

combined recovery/recrystallisation process. The appearance of the rotated cube and $\{111\}\langle 110\rangle$ components in the microtecture are also not represented in the macrotexture.

The data were analysed for correlation between proportions of the true recrystallised components-cube, Goss and rotated cube, CGR,- and position. Figure 7 shows the proportion of CGR components per crack (for cracks four or more grains in length) where the cracks are numbered ascendingly according to their distance from the start of the primary crack. The average proportion of CGR components is $37 \%$. It is evident from Figure 7 that the CGR proportion per crack is randomly distributed with respect to distance along the primary crack.

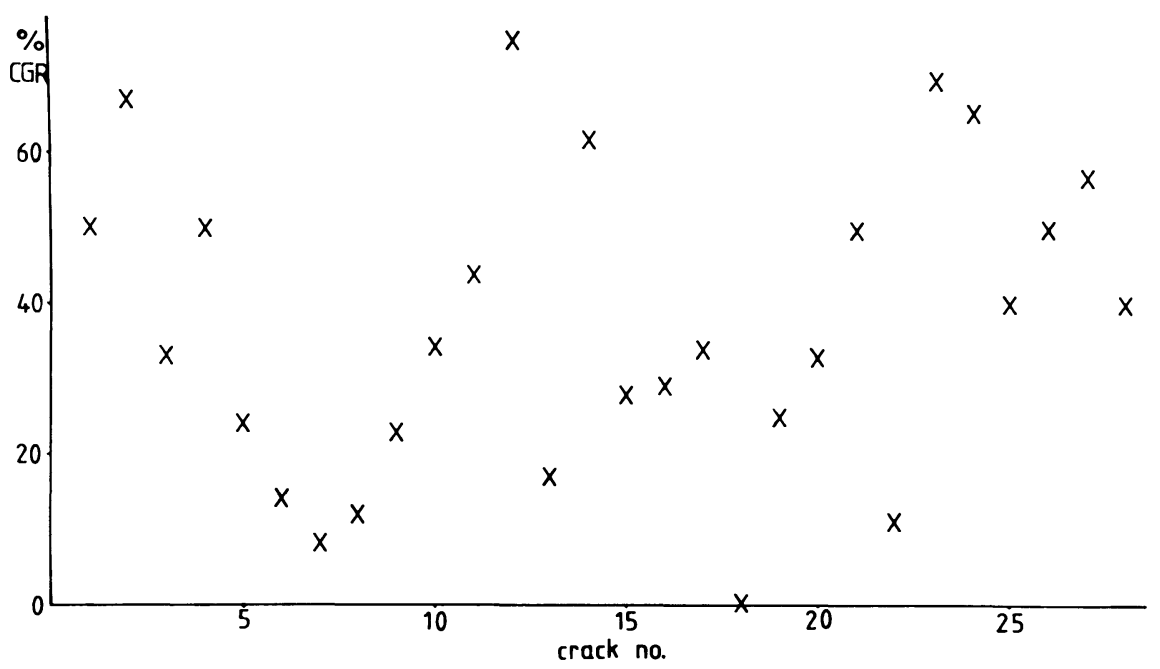

Figure 7 Proportions of "recrystallised texture" components, i.e. cube, Goss and rotated cube (CGR) in each side-branched crack where they are numbered according to their proximity to the start of the primary crack. 


\section{DISCUSSION}

The EBSD measurements have shown that the texture of this material is inhomogeneous, and there are differences between both the cracked and control sample populations and also between individual control samples. Since the macrotexture shows a large proportion of Goss grains, presumably there exist other regions of microtexture where the high S-texture populations recorded in the current data are compensated for by high Goss so that the sum of all microtextures accurately reflects the measured macrotexture.

The most significant feature of the data is the relative occurrence of the four symmetry related forms of the S-component. It is therefore pertinent to discuss the S-component in more detail. If the S1 component is designated as (123)[412] ], the misorientation between it and the other three crystallographic equivalents, S2, S3, S4 are:

where $S 1$ is (123)[41ㄹ]

$\mathrm{S} 2$ is $(213)[14 \overline{2}]$

S3 is $(213)[\overline{1} 42]$

$\mathrm{S} 4$ is $(123)[\overline{4} \overline{1} 2]$

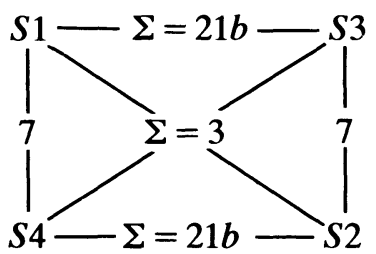

Hence both a mirror relationship $(\Sigma=3)$ and the mobile $40^{\circ} / 111$ relationship (near $\Sigma=7$ ) exist between various combinations of the four variants. The positions of the four variants on 111 pole figures, and also the configuration of an EBSD pattern for each variant, is shown in Figure 8. It is clear that the four variants can be distinguished unambiguously by EBSD.

The two mirror-related S-variants observed in the control areas could result from two shear bands of different orientation. A second possibility is that some areas have undergone "extended recovery" (Orsund and Nes, 1989), also referred to as in-situ or continuous recrystallisation, which implies that the rolling texture is retained.
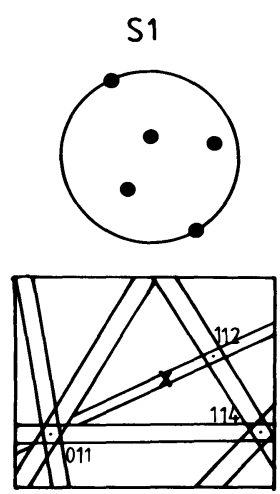
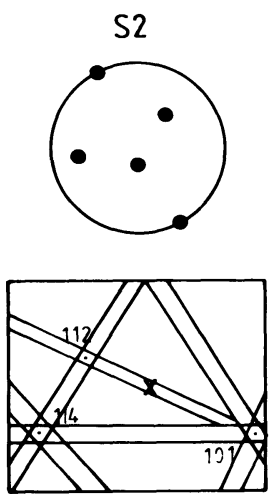
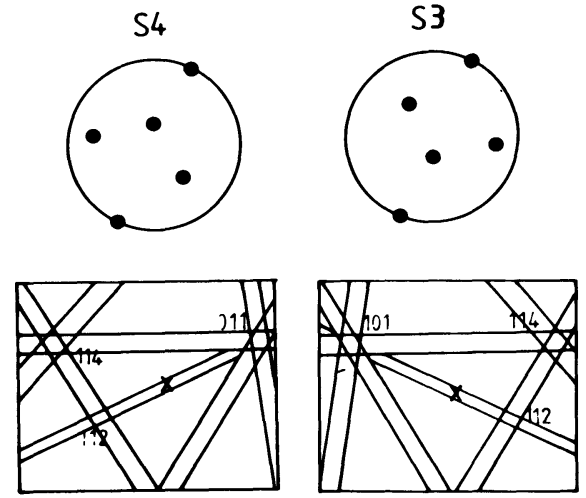

Figure 8 The location of the four S-texture variants, (a) S1, (b) S2, (c) S3 and (d) S4 on 111 pole figures, each accompanied by a corresponding schematic EBSD pattern. The pattern centre is marked $X$ and the rolling direction (RD) is horizontal in the pattern. 
Extended recovery has been previously observed in $8090 \mathrm{Al}-\mathrm{Li}$ sheet and associated with slow heat-up rates (Bowen, 1990). In the present work "classical" recrystallisation might be characterised by one $S$ variant and a second, mirrorrelated variant could exist in a continuously recovered region. Mirror-related areas of S-texture have been previously observed in pure aluminium between deformed and recrystallised areas of microstructure (Hjelen and Nes, 1988).

The deformation characteristics for each S-variant are different, and this provides one reason for the erratic crack paths observed. Strain accommodation is therefore easier for a single $\mathrm{S}$ variant than for a mixed distribution as observed in the cracked area. Deformation induced by the primary crack has produced an adjacent microstructure where slip cannot readily occur because of the mixed $S$ components and secondary cracking is therefore initiated instead. Clearly more work is warranted in this area.

\section{CONCLUSIONS}

1. The application of electron back-scatter diffraction to an $8090 \mathrm{Al}-\mathrm{Li}$ sheet alloy has clearly shown positional variations in microtexture on a linear scale of the order of 50 grains, and furthermore these microtextures do not match the averaged macrotexture indicating an inhomogeneous distribution of orientations. The S-texture is the majority component in the microtextures, whereas the overall macrotexture is predominantly the Goss component.

2. The microtexture of grains associated with secondary branched fatigue cracks is different from that of "control" grains which are on the same plane of polish within the specimen. The principal difference between the cracked and control grains is that a single variant of the S-texture, either S1 or the mirror related variant $S 2$, dominates the control microtexture whereas all four $S$-texture variants are present in the cracked grains data set.

3. The mirror-related variants of the S-texture occur in spatially separate clusters in the control grains, and some grouping of related orientations is evident.

\section{ACKNOWLEDGEMENTS}

The author is grateful to Alcan International for provision of specimens, to Dr. K. Gatenby for many useful discussions and to Dr. A. Bowen for his comments.

\section{REFERENCES}

Bowen, A. W. (1990). The influence of heating-up rate on recrystallisation in $8090 \mathrm{Al}-\mathrm{Li}$ alloy sheet. Proc. Recrystallisation '90', Ed. T. Chandra, Min. Met. Mat. Soc., USA, 157-162.

Bowen, A. W., Gatenby, J. M., McDarmaid, D. S. and Palmer, I. G. (1992). Influence of Recrystallised Grain Structure and Texture on the Mechanical Properties of Damage Tolerant 8090 $\mathrm{Al}$ - Li Sheet. Proc. 6th Int. Al-Li Conf., Garmisch-Partenkirchen, Germany, DGM Informationgesellschaft $\mathbf{m b H}$. 
Bunge, H. J. and Weiland, H. (1988). Orientation Correlation in Grain and Phase Boundaries. Tex. and Mic. 7, 231-263.

Dingley, D. J. and Randle, V. (1992). Microtexture Determination by Electron Back-Scatter Diffraction (Review). J. Mat. Sci., 27, 4545-4566.

Frank, F. C. (1988). Orientation Mapping. MRS Bull., March, 24-29.

Hjelen, J. and Nes., E. (1988). The Evolution of Recrystallisation Textures in Aluminium as Examined by Electron Back Scattering Patterns. ICOTOM8, edited by J. S. Kallend and G. Gottstein, pp. 597-602. USA: The Metallurgical Press.

Orsund, R. and Nes, E. (1989). Subgrain growth during annealing of heavily deformed metals. Scripta Met., 23, 1187-1192.

Randle, V. (1990). Representation of Grain Misorientations (mesotexture) in Rodrigues-Frank Space. Proc. Roy. Soc. Lond., 431A, 61-69.

Randle, V. (1992). Microtexture Determination and its Applications, London: Institute of Materials.

Weiland, H. (1992). The Determination of Long-Range Misorientations in the Microstructure of Materials, Acta Met. Mat., in press. 